\section{Transición en la concepción de la viabilidad de prematuros extremos: análisis sociodiscursivo}

\author{
A shift within the notion of extreme \\ prematurity viability: a social discursive \\ analysis
}

\section{Resumen}

El objetivo de este trabajo es mostrar cómo en nuestro país se está transitando hacia una nueva concepción de la viabilidad de la prematuridad extrema (recién nacidos con menos de 28 semanas de gestación), caracterizarla y contextualizarla.

Para ello, se utilizan datos procedentes de una investigación (P.N. I+D+i, CSO20II-24294) que ha obtenido resultados válidos sobre el desarrollo de I.200 nacidos con I. 500 gramos o menos al nacer. mediante encuesta poblacional multimétodo, y producido cuatro grupos de discusión y 25 entrevistas abiertas con agentes cualificados. Se recogen aquí los resultados del análisis sociodiscursivo del material cualitativo centrado en esta cuestión y del estudio de diversos documentos sobre viabilidad en la prematuridad extrema.

En cuanto a los resultados, en España se viene transitando de manera más práctica que consciente desde una concepción reduccionista de la prematuridad extrema, ligada al normativismo de la bioética y al aislamiento del recién nacido, a una mirada más ensamblada, compleja y heterogénea, acorde con la vigente renovación de la cultura, la práctica y la política biosanitaria en nuestro país.

\section{Palabras clave:}

Viabilidad; prematuridad extrema; toma de decisiones clínicas; limitación del esfuerzo terapéutico; problemas bioéticos.

\begin{abstract}
The aim of this paper is to show that in Spain there is a shift to a new notion of viability of extreme prematurity (babies born with less than 28 weeks of gestation) as well as its features and contexts.

The results from a national research project (P.N. I+D+i CSO2OI I-24294, ) that implemented a survey on I 200 newborn with $\leq \mathrm{I} 500 \mathrm{~g}$., as well as four discussion groups and 25 indepth interviews with involved agents. This paper summarizes the main results of the socio discursive analysis of the qualitative material as well as analysis of several documents on the viability of extreme prematurity.
\end{abstract}

With regards to the results there seems to be an ongoing transition, more practical than conscious, from a reductionist notion of extreme a bioethical normative thinking and the newborn isolation, to a more assembled, complex and heterogeneous point of view. The than a conscious approach.

This new notion of viability is an integral part of the ongoing renewal in the bio-sanitary culture, practice and politics in Spain.

\section{Keywords}

Viability; extreme prematurity; clinical decision-making; withdrawing treatment; bioethical issues. prematurity viability, linked to transition is lead by a practical rather

\author{
Fernando J. García Selgas \\ <fgselgas@cps.ucm.es> \\ Universidad Complutense de Madrid
}

Concepción Gómez Esteban <conchagomez@cps.ucm.es>

Universidad Complutense de Madrid

\section{Belén Sáenz-Rico de Santiago \\ <bsaenzri@edu.ucm.es>}

Universidad Complutense de Madrid

Para citar:

García, F.J. et al. (2016): "Transición en la concepción de la viabilidad de prematuros extremos: análisis sociodiscursivo", Revista Española de Discapacidad, 4 (I): 67-8I.

Doi: <http://dx.doi.org/IO.5569/23405 IO4.04.OI.04>

Fecha de recepción: 19-02-20I6 Fecha de aceptación: 06-06-2016 
A José García Hortelano y a Jesús Pérez Rodríguez, grandes pediatras y hombres de bien.

Introducción

El parto prematuro es actualmente uno de los principales problemas de salud. Según datos de la Organización Mundial de la Salud (OMS, 20I 5), se estima que cada año nacen unos I 5 millones de niños antes de que se cumplan las 37 semanas de gestación, una cifra que va en aumento. Aunque más del $60 \%$ de los nacimientos prematuros se producen en África y Asia meridional, el parto pretérmino y sus consecuencias constituyen un verdadero problema mundial porque las complicaciones relacionadas con la prematuridad son la principal causa de defunción en los niños menores de cinco años y provocaron en 2013 cerca de un millón de muertes, si bien las tasas de supervivencia presentan notables disparidades entre los distintos países del mundo (OMS, $2015)^{\mathrm{I}}$.

En el caso de España, en los últimos años el porcentaje de nacimientos prematuros representaría en torno al $6,5 \%$ del total de alumbramientos según datos del Instituto Nacional de Estadística (INE), cifra que los indicadores del Sistema Nacional de Salud (SNS) elevan en más de un punto, dado que estos organismos públicos operan de manera diferente a la hora de establecer el número de partos pretérmino que se producen en nuestro país. Si atendemos a los datos relativos a los prematuros extremos, estos representarían algo menos del $0,3 \%$ del total de nacidos cada año.

Muchos de los bebés que nacen prematuramente y sobreviven sufren algún tipo de discapacidad de por vida, derivada principalmente de la inmadurez orgánica que presentan. Las

I. En los I 84 países estudiados por la OMS (2015), la tasa de nacimientos prematuros supone entre el $5 \%$ y el i $8 \%$ de los recién nacidos, siendo en los países de ingresos bajos una media del I $2 \%$ frente al $9 \%$ en los países de ingresos más altos. principales discapacidades que puede desarrollar este colectivo están relacionadas con el aprendizaje, la comunicación, la movilidad y los problemas de tipo visual y auditivo, lo que se produce en mayor medida en los recién nacidos a los que nos referiremos en este artículo: los bebés "prematuros extremos", esto es, nacidos con una edad gestacional (EG) de $<28$ semanas, y especialmente aquellos que nacen entre la semana 22 y 26 de gestación. Por ello, "la disminución de la mortalidad sin aumentar la morbilidad y sus secuelas constituye uno de los retos más importantes de la medicina perinatal, sobre todo en el grupo de menor edad gestacional” (GarcíaMuñoz et al., 20I4) y es crucial que todos los niños nacidos muy prematuramente tengan un adecuado seguimiento sociosanitario y educativo, que permita minimizar las eventuales secuelas que pudieran padecer (Gómez, 20I4; Gómez et al., 2OI4).

En buena medida la cuestión de la viabilidad en la prematuridad extrema (VPE), esto es, establecer límites, criterios y procedimientos de gestión de supervivencia de estos neonatos, y sobre todo de los nacidos entre la semana $22 \mathrm{y}$ 26 de EG, es una de las principales maneras de responder a ese reto de la medicina perinatal. La VPE ha sido siempre una cuestión compleja, debido a las responsabilidades que involucra, las fuertes emociones que moviliza y la diversidad de factores que pueden intervenir en ella. Pero a lo largo de los últimos decenios se ha ido haciendo más compleja al involucrar a más agentes (comités de ética, psicólogos, asociaciones de padres, etc.), por los rápidos desarrollos de la biotecnología (ecografías, catéteres epicutáneos, surfactante, resonancias magnéticas del cerebro, etc.) y los acelerados cambios socioculturales que se han ido produciendo durante este tiempo (importancia creciente de las asociaciones de afectados, medidas diversas de humanización, implantación del modelo de cuidados centrados en la familia, etc.).

La rapidez y profundidad de estos cambios han hecho que resulte difícil racionalizarlos, expresarlos discursivamente o mantenerlos en una relación coherente con el marco normativo (moral y legal) en el que necesariamente se 
integran. A esa dificultad han contribuido el que la determinación de la VPE sea uno de los elementos claves en un tema tan polémico como el aborto (Cabero, 2009: 67) y el que constituya un caso ejemplar del modo en que hoy se ejerce el poder en las sociedades democráticas, como biopoder. Efectivamente, una vez que el poder se concibe no solo como represión o control, sino también como potenciación y capacitación y que entendemos que los conflictos de intereses por redefinir los mapas de (im)posibilidades son los que hoy definen las cuestiones políticas en las democracias (Mouffe, I999), podemos aceptar la tesis de que actualmente el ejercicio del poder en los países desarrollados no gira tanto en torno a "hacer morir" o "dejar vivir", a la muerte, como sucedía hasta el siglo XIX, cuanto en torno a "hacer vivir" o "dejar morir", a administrar la vida (Foucault, I976), en la que la perinatal es un caso paradigmático (Weir, 2006).

Sea por la razón que sea el caso es que, como constatamos con este trabajo, las rápidas transformaciones en las prácticas biosanitarias referidas a la VPE han pasado de afrontarla inicialmente como una cuestión de pronóstico (estocástico) clínico a la que, en algunos casos, se añaden algunas consideraciones de tipo ético (esto es, afrontarla desde un planteamiento bioético), a admitir su complejidad y multidimensionalidad y empezar a dar voz y agencia a padres, personal de enfermería y comunidad social (recursos, educación, etc.), en lo que sería un planteamiento bio-político².

2. Entendemos por planteamiento bioético el que asume que la incertidumbre de determinadas decisiones clínicas se resuelve sobre la base de un diagnóstico y un pronóstico (en este caso respecto a la VPE) que se complementan con una reflexión ética que, regida por el respeto a la dignidad de las personas (bebés, sanitarios, familiares, etc.), intenta armonizarlos con los valores hegemónicos implicados (vida del bebé, no-maleficencia, justicia, no-discriminación, etc.). Es un planteamiento visible en Aguayo et al. (20I I: 9-I3 y 77-80) o en Moreno (2015: 247).

Planteamiento biopolítico sería aquel que, en lugar de aislar la situación del prematuro, la ve inserta en una multiplicidad de espacios biosociales, empezando por la madre y llegando a las políticas reproductivas del Estado. No parte de una separación radical entre lo biológico o natural y lo sociopolítico o cultural, de modo que el establecimiento de lo posible o imposible en la vida (en este caso del bebé prematuro), la especificación de qué vidas son socialmente valiosas y la disputa por
Sin embargo, y a pesar de que este nuevo y más complejo planteamiento se va consolidando en el día a día de las Unidades de Cuidados Intensivos Neonatales (UCIN) de nuestro país, en muchos estudios y propuestas de protocolos referidos a la atención y seguimiento de la VPE da la impresión de que cuesta mucho ponerlo en palabras y en valor. Por ello, el objetivo de este artículo es mostrar, a partir del análisis de los discursos de los principales agentes implicados en esta cuestión y del estudio de diversos documentos de referencia, la emergencia práctica de esta nueva concepción de la VPE y cuáles son sus rasgos más importantes, así como su conexión con la vigente recomposición de la realidad biosanitaria.

\section{Metodología}

El material empírico sobre el que se sustenta este trabajo procede de un proyecto financiado por el Plan Nacional de I+D+i (ref. CSO2O I I24294), centrado en los nacidos con $\leq \mathrm{I} 500 \mathrm{~g}$. entre I993 y 20 I I en España, cuyos objetivos eran identificar los principales problemas biopsicosociales que presenta esta población y mostrar la relación de estos problemas con las características y practicas familiares y con los cambios producidos durante ese período en el tratamiento sociosanitario de estos menores ${ }^{3}$.

establecer unos valores u otros como hegemónicos vendrían a constituir las políticas de la vida o bio-políticas en las que se encuentran inextricablemente enredadas prácticas tecnocientíficas, estrategias de poder y formas de (auto)producción de sujetos 'saludables', 'autónomos', etc. (Lemke, 20 I I: I-2 y II 8-223; Rabinow y Rose, 2006: I97-I98). Esta distinción es más bien una distinción analítica, para marcar dos puntos distintos y distantes del desplazamiento habido en las prácticas biomédicas involucradas en la VPE. Es posible, por ello, que en determinados casos se aproximen o incluso se expresen en un mismo documento, por ejemplo, la concepción biopolítica parece asomarse tímidamente tras las consideraciones de Aguayo et al. (20II: I I2-I I3) sobre la atención centrada en la familia.

3. Este proyecto obtuvo desde sus inicios el apoyo de tres entes promotores observadores (EPOs): la Sociedad Española de Neonatología, el Comité Español de Representantes de Personas con Discapacidad y la Asociación de Padres de Niños Prematuros, que se han implicado activamente a lo largo del 
Si bien el estudio de los procesos relacionados con la viabilidad de los prematuros extremos no era un objetivo explícito de este trabajo, esta cuestión surgió en repetidas ocasiones a lo largo del desarrollo del proyecto, especialmente en el trabajo de campo cualitativo, constituyéndose como un tema al que el análisis sociodiscursivo de entrevistas y grupos de discusión debía de atender y que obligaba a explorar otros materiales adicionales.

Para obtener los objetivos planteados se han utilizado diferentes métodos de análisis y técnicas, desde la explotación de fuentes secundarias a la producción de datos cuantitativos y cualitativos. El trabajo de campo ha consistido, por una parte, en la realización de una encuesta a familias que ha recogido datos válidos sobre el desarrollo de $\mathrm{I} 200$ nacidos con SI 500 g. utilizando la tecnología CATI y la tecnología CAWI (el grupo de investigación creó su propio sistema CAWI de encuestas online basado en software de libre distribución). Para el análisis de los datos se utilizó el programa SPSSWIN, versión I9 y, previamente, se reequilibró la muestra a partir de las variables "peso al nacer" y "año de nacimiento", utilizando los datos proporcionados para este proyecto por el INE. Antes de lanzar la encuesta, se había realizado un amplio trabajo cualitativo, que incluía observación no participante en algunas Unidades de Cuidados Intensivos Neonatales, cuatro grupos de discusión y 25 entrevistas en profundidad en Madrid y Sevilla. La técnica empleada para analizar los datos cualitativos ha sido el análisis sociodiscursivo. Sobre él se basa el presente artículo. Por eso conviene que detallemos un poco más el modo en que lo hemos realizado.

El proceso del análisis sociodiscursivo se estructuró en dos fases: en la primera, el equipo de investigación realizó un análisis del material producido por las entrevistas y los grupos de discusión cruzando, ligando,

desarrollo del proyecto. También se ha beneficiado de la colaboración establecida con diversos hospitales de referencia en la atención a grandes prematuros y con entidades y profesionales que trabajan en este ámbito. comparando y estudiando el discurso desplegado por los entrevistados, que habían sido escogidos por pertenecer a los principales ámbitos involucrados en esta cuestión: unidades/servicios de neonatología, familias, servicios de seguimiento sociosanitario y medio educativo. Los campos semánticos, las contradicciones, los desplazamientos semióticos, las metáforas dominantes, las autocorrecciones o matizaciones según avanzaba la entrevista, o según los grupos se centraban en temas controvertidos, y el señalamiento de imágenes o tesis supuestas fueron los recursos principales del discurso que nos ayudaron a detectar los nudos temáticos que de forma entrelazada, pero diferenciada, marcaban en cada caso las distintas aproximaciones a la viabilidad de la prematuridad extrema. En una segunda fase, el equipo desentrañó y analizó esos nudos temáticos, cruzó y comparó los discursos de los distintos agentes involucrados y estudió otros documentos que completaban el espacio discursivo establecido en torno a esta cuestión. El análisis realizado nos permitió constatar que estamos inmersos en la transición ya mencionada y señalar algunos de sus rasgos característicos, como haremos a continuación.

\section{Resultados}

\section{Estado de la cuestión: los hechos}

Para que el análisis sociodiscursivo y la exposición de sus resultados no se pierda en el círculo infinito de las interpretaciones de discursos (el círculo hermenéutico), resulta conveniente empezar recogiendo de la manera más objetiva posible algunos datos y planteamientos sobre la VPE publicados por los expertos sanitarios más directamente vinculados con la atención a estos neonatos. A continuación, recordaremos el marco legal pertinente, así como algunos rasgos actuales de las prácticas sanitarias relativas a la VPE. De aquí pasaremos, en el siguiente apartado, a los resultados de nuestro análisis. 


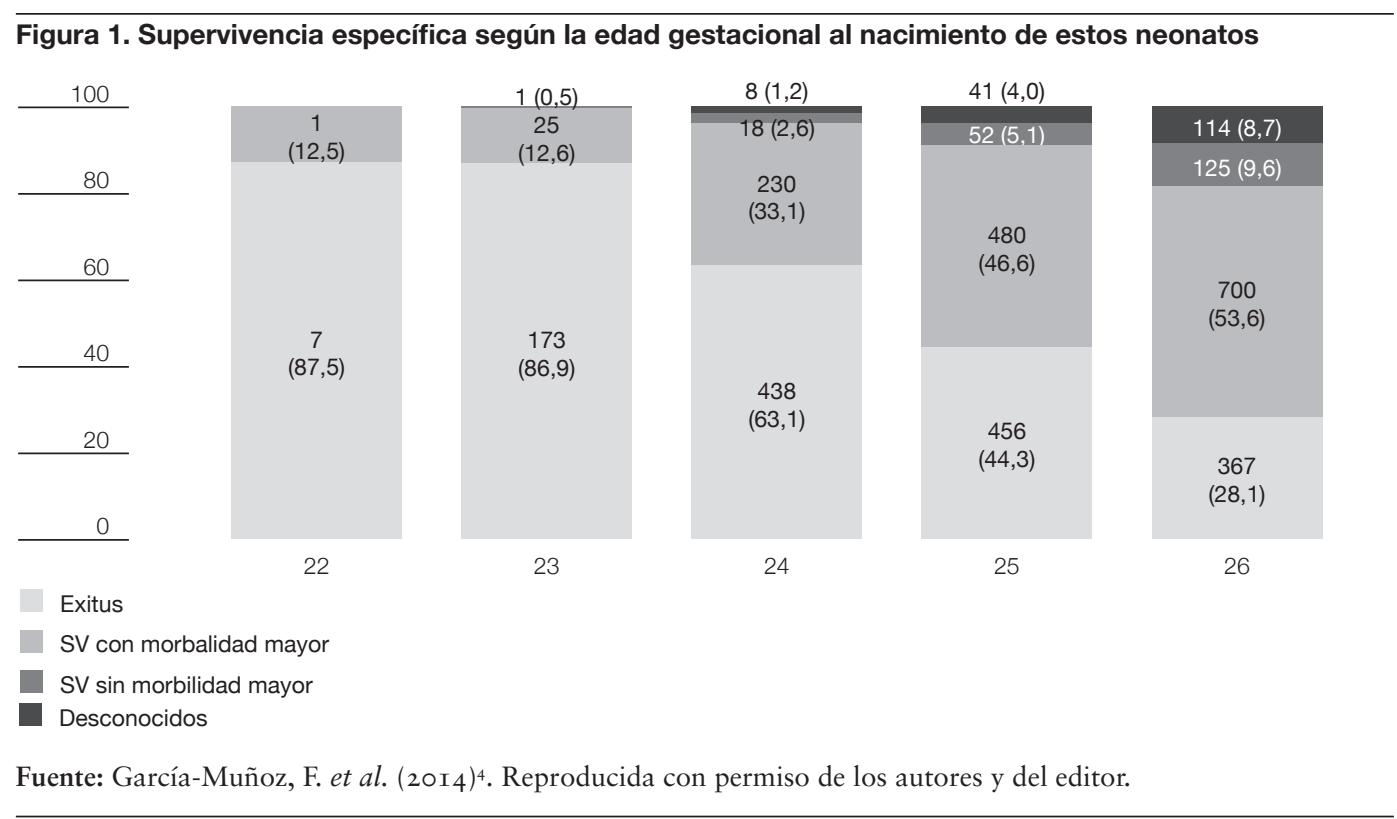

Los análisis realizados a nivel internacional ponen de manifiesto que los datos sobre VPE varían de unos países a otros, de unas regiones a otras e, incluso, de un hospital a otro (Saigol y Doyly, 2008; García-Muñoz et al., 20I4). Para nuestro caso, tomaremos como referencia el estudio poblacional de la VPE de nacidos con $\leq 26$ semanas en España entre 2004 y 2010 y cuyos resultados se resumen perfectamente en la Figura I, que muestra la supervivencia específica con y sin morbilidad mayor ${ }^{5}$ según la EG al nacimiento de estos neonatos:

4. Datos referidos a recién nacidos ingresados vivos en las UCIN de la red de seguimiento neonatal SEN I 500 , con un peso medio que va de $600 \mathrm{~g}$. con 22 semanas de EG a 864 g. con 26 semanas; previamente habían fallecido en la sala de partos el 74,2\% de los que nacieron con 22 semanas, el $23,8 \%$ de los que lo hicieron con 23 semanas, el $4,7 \%$ de los de 24 semanas, el I, $5 \%$ de los de 25 semanas, y el $0,5 \%$ de los de 26 semanas de EG.

5. Por morbilidad mayor se consideró en este estudio la presencia de uno o más de los siguientes problemas: hemorragia intraventricular (HIV) grave (grados 3 y 4 de Papile); lesión de la sustancia blanca: leucomalacia periventricular quística y/o hiperecogenicidades persistentes; displasia broncopulmonar (DBP) definida como dependencia de oxígeno a las 36 semanas de edad posmenstrual; enterocolitis necrosante (NEC) $\geq$ estadio 2 de Bell; sepsis bacteriana temprana y/o meningitis (cultivo positivo $<72 \mathrm{~h}$ ) o sepsis y/o meningitis tardía (cultivo positivo $>72 \mathrm{~h}$ ), y retinopatía de la prematuridad (ROP) $\geq$ grado 3, y/o tratamiento mediante laserterapia.
En relación con los datos recogidos en la Figura I, hay que señalar que, pese a que en nuestro país en los últimos años se ha producido un importante incremento de nacidos muy prematuramente (con $<32$ semanas de EG) y de recién nacidos de muy bajo peso, la tasa de supervivencia de estos bebés ha experimentado una mejora substancial, especialmente desde 2005 (Hernánde et al., 2005; Guzmán et al., 20I3; García et al., 20I3; García-Muñoz et al., 20I4), aunque las morbilidades no han disminuido en la misma medida, especialmente en el colectivo de prematuros extremos en los que se centra este artículo (Sánchez Luna 20I4; Ochiai et al., 20I4).

La evolución producida en España en relación con la supervivencia y las morbilidades mayores de los niños nacidos muy prematuramente es similar a la experimentada por otros países como Inglaterra, Suecia o Australia en los que, aunque el aumento de supervivencia se acompañó de una reducción de ese tipo de morbilidades, no sucedió lo mismo en el caso de los neonatos que nacieron con menor edad gestacional 6 .

6. Por ejemplo, en Inglaterra la supervivencia de los prematuros extremos aumentó un I3 \% entre I 995 y 2006, a la vez 
Por último, según nuestra encuesta, el porcentaje de niños nacidos con < 500 g. entre I993 y 20 I I que tienen morbilidades es el 44,2 \%. Se trata de un porcentaje muy superior al que encontramos en la población total de menores (solo del I,9\% según datos de INE/MSPS, 2008), aunque a lo largo de este período se aprecia una disminución de las morbilidades graves y permanentes, incluso en el caso de los nacidos prematuramente con menor peso. Nuestra encuesta muestra también que los factores que en mayor medida explican la existencia de morbilidades en estos niños son de carácter genético y perinatal, por lo que se entiende perfectamente la carga de responsabilidad que entrañan las decisiones y prácticas clínicas que tienen lugar al nacimiento y en el tratamiento hospitalario de estos neonatos.

El marco legal inmediato para la VPE en nuestro país se extrae de la Ley Orgánica 2/20I0, modificada por la Ley Orgánica I I/20I 5 de 2 I de septiembre ${ }^{7}$, que en su Título II despenaliza la interrupción voluntaria de la gestación hasta la semana 22 por "graves riesgos para la vida o la salud de la madre o el feto" y a partir de ese momento solo cuando se detecten anomalías incompatibles con la vida. Sobre esta base, y adaptando con ciertas diferencias (Salguero García, 20I3), la propuesta de la International Liaison Committee on Resuscitation, la Sociedad Española de Neonatología (SENeo) recomienda no iniciar la reanimación con EG de menos de 23 semanas $\mathrm{o}<400 \mathrm{~g}$. de peso, pero sí iniciarla cuando el neonato nazca con $\geq 25$ semanas de EG (Iriondo et al., 20I I), lo cual genera una "zona de penumbra" de la VPE en las 23-24 semanas de EG, en la que el criterio "estimaciones de probabilidad de supervivencia" (Cole et al., 2010; Manktelow et al., 2013) ayuda a plantear la cuestión, pero no ayuda a

que disminuyeron las morbilidades mayores en un I 2, $5 \%$ para los nacidos con EG de 24 y 25 semanas; en cambio, esto no sucedió en el caso de los de 22 y 23 semanas de EG, con un $-0,4 \%$ de supervivencia y solo un 2,5\% de disminución de morbilidades (Moor et al., 20I2).

7. Ambas leyes, sin embargo, se sitúan bajo un paraguas más amplio de normas jurídicas, como la Constitución Española o la Carta Europea de los Derechos del Niño (ver Aguayo et al., 2OII: I7-23 y I 55). resolverla y, por ello, se ve (Iriondo et al., 20I I) la necesidad de tener en cuenta la respuesta y estado individual del neonato, además de la opinión parental.

Para recoger algunos rasgos de las prácticas sanitarias efectivas en relación con la VPE, en especial lo que sucede en esa "zona de penumbra", podemos volver a los datos proporcionados por el estudio de García-Muñoz et al., (20I4: 355). En él, se recoge que de los exitus (fallecidos) contabilizados en la Figura I se declaró alguna forma de adecuación del esfuerzo terapéutico "en un 35,9\%, siendo más frecuente entre los de menor edad gestacional: 42,9\% (22 semanas), 5 I, I \% (23 semanas), 39, I \% (24 semanas), 33,2 \% (25 semanas) y $28,8 \%$ (26 semanas)". Esto nos puede llevar a plantearnos dos cuestiones respecto a las expectativas que se tenían sobre estos exitus al comienzo del proceso: primera, cuántos se esperaba que fallecieran, pero no hemos encontrado datos específicos para responderla más allá de su propia afirmación (ibíd.) de que "la supervivencia de niños menores de 23 semanas de EG en nuestro medio es excepcional" ${ }^{\text {; }} y$, segunda, cuántos se esperaba que fueran a tener morbilidades graves, respecto de lo cual resaltan (ibíd.) que "las posibilidades de supervivencia sin morbilidad mayor en recién nacidos de 23 y 24 semanas de EG son escasas", a lo que se añade (ibíd.) que "los padres deberían participar, cuando sea posible y después de un apropiado consejo no directivo, en la toma de decisiones". Volveremos sobre todo esto más adelante, al analizar los discursos.

La crudeza y complejidad que envuelve estas decisiones se extiende en buena medida al resto de las prácticas sanitarias relativas a la VPE. Se observa, por ejemplo, que los profesionales en las UCIN se enfrentan de manera cotidiana a

8. Se puede acudir a datos de hospitales concretos como el Hospital Universitario La Paz de Madrid, que es de nivel terciario. En un detallado informe sobre la mortalidad infantil se dice que de aquellos a los que se les aplicó la LET el 83,9\% fue clasificado como fallecimientos esperados e inevitables, a lo que más adelante añaden que "en nuestra serie $[. .$.$] la$ prematuridad extrema y sus secuelas es la primera indicación de esta decisión” (Alonso et al., 20I 2: 347). 
cuestiones en torno a la limitación del esfuerzo terapéutico y que, como señala Caserío (20I4), es frecuente que se precise más de una discusión en el equipo antes de hacer la propuesta a los padres de este tipo de práctica. Pueden hacer falta hasta tres sesiones para proponer a los padres la adecuación del esfuerzo diagnóstico y terapéutico (AEDT) (en el estudio de Caserío, tras la primera propuesta la AEDT la acepta un 7I,4 \% de los padres; un IO,2 \% tras la segunda y $\%$ en la tercera).

En resumen, se admite que la VPE se establece sobre la base de un pronóstico médico de las posibilidades de supervivencia sin morbilidades graves para el recién nacido. Sin embargo, parece que la gestión concreta de esa viabilidad y el eventual establecimiento de un límite general ha tendido a realizarse de manera efectiva en el cruce desigual de capacidades biomédicas, reflexiones éticonormativas y el reclamo de la participación de la familia. Lo cual nos va situando, y más aún con las nuevas prácticas que se han ido instalando en las UCIN en la última década, ante una situación bastante más compleja y dinámica de lo que es capaz de manejar la concepción que hasta hace poco ha venido subyaciendo a esta cuestión y que presentaba esos ámbitos confluyentes como separados, distintos y distantes, y al recién nacido como una figura individual y prácticamente aislada. El análisis de los discursos de los principales agentes implicados en esta cuestión nos va a permitir mostrar mejor cómo, de hecho, se está produciendo un desplazamiento hacia una perspectiva capaz de apreciar la interconexión interna y práctica de esos ámbitos y de percibir la VPE como parte y resultado de ella.

\section{Análisis de los discursos}

I. El análisis del discurso desplegado en los documentos que la SENeo toma como referencia para la viabilidad (Iriondo et al., 20II) muestra tres nudos temáticos sobre los que se organiza su concepción: ambigüedad o tensión de la viabilidad en la prematuridad extrema, (des)agregación de sus ingredientes y re-conceptualización. Al deshacer dichos nudos mediante el análisis sociodiscursivo se pone de manifiesto que, aunque inicialmente subyace en los planteamientos un modelo clásico (cartesiano: individualista y dualista, como veremos más abajo), según avanza el discurso se va dando paso a otra concepción más compleja que no responde a esas características.

Está bastante generalizado en descripciones clínicas (García-Muñoz et al., 20I4; Sánchez Luna, 2014) y en propuestas éticas (Jiménez y Molina, 2008; Raspall y Royes; 2009) presentar la viabilidad en la prematuridad extrema como una realidad ambigua o más bien tensa por la colisión entre las posibilidades terapéuticas, el compromiso hipocrático, los intereses del recién nacido, las expectativas de la familia y el coste-beneficios del cuidado. Con ello se asume una mirada que establece compartimentos estancos para cada uno de los ingredientes de esta toma de decisiones y sitúa al recién nacido como centro silente y aislado del proceso, separado de esos compartimentos. Por ejemplo, en algunas reflexiones bioéticas como las de Gladys et al. (2004), se presenta el conflicto del neonatólogo ante una incierta viabilidad en la prematuridad extrema como resultado de la tensión que se produce entre conjuntos disjuntos de obligaciones y ámbitos: el ético, el clínico, el familiar y el social.

La presencia reiterada de expresiones como "se debe", "hay que”, "es necesario", "dignidad humana”, etc. y la referencia a un supuesto conjunto de valores, no son simples marcas de un discurso normativo, sino que se asumen como rasgos de una lógica específica que cierra el ámbito de la (bio)ética sobre sí mismo y por ello exige la existencia de especialistas en él. Paralelamente, el juramento hipocrático, el conocimiento y los recursos tecnocientíficos acotarían el ámbito médico; mientras los afectos y emociones definirían el mundo familiar del neonato; y la distribución de 
posibilidades y el uso de recursos el ámbito social. Por ello, las recomendaciones (Raspall y Royes, 2009) o las descripciones (Saigol y Doyly, 2008) se hacen separadamente, a continuación una de otra, implicando muchas veces un cierto orden jerárquico que da prioridad a unos ámbitos (normalmente el médico) sobre los otros.

Y lo más curioso es que el recién nacido no termina de entrar en ninguno de esos ámbitos y se le concibe como un ser u organismo individual separado de ellos. Es más, cuando la bioética (Jiménez y Molina, 2008) propone "la pérdida de capacidad cognitiva y de comunicación" como criterio de no viabilidad, lleva al extremo ese aislamiento, circunscribiéndolo a la mente, en un ejercicio supremo de cartesianismo dualista y mentalista. Esta concepción, que aísla al prematuro extremo de lo que le rodea, es la que subyace a la pretensión de que es el equipo médico el que representa y defiende "los intereses del recién nacido" (Jiménez y Molina, 2008): una vez separado (imaginaria, conceptual y físicamente) de la madre que lo engendra y de la sociedad que lo nutre y hace posible, el recién nacido queda como flotando en un nuevo éter (¿el éter de la biotecnología?), esperando a que un agente externo, que se pretende totalmente desinteresado y, por ello, objetivo, venga a hablar en su nombre, a defender sus intereses.

Lo anterior no impide que se pueda percibir también en estos discursos la paulatina asunción de una concepción bien distinta, en la que las lógicas de esos ámbitos aparecen como interrelacionadas y mutuamente constituidas. Por ejemplo, cuando diversos documentos (Gálvez, 2006; Pi-Sunyer, 20I3) ponen de relieve la dureza emocional (inseguridad, tristeza, soledad, dolor) que supone para el personal sanitario todo lo relativo a la VPE, se abre la puerta a admitir que la labor clínica también está atravesada por lógicas emocionales y afectivas, que la ponen en directa relación con el ámbito familiar.
Otro tanto sucede cuando la VPE se hace depender directamente de la distribución sociopolítica de recursos (Sánchez Luna, 20I4) y la calidad asistencial de los hospitales que atienden a estos neonatos, o de los procesos de apoyo y seguimiento que se establecen, de modo que la VPE y la existencia misma del recién nacido se van percibiendo como parte y efecto del ensamblaje de estos ámbitos confluyentes. Las reiteradas llamadas (Iriondo et al., 20I I) a un cierto tipo de decisión compartida con los padres y determinado consenso ético o legal, con especial insistencia en la "zona de penumbra” -que es donde estaría de facto el límite de la viabilidad en la prematuridad extrema-, sitúan la viabilidad de estos neonatos en la encrucijada común de lógicas y ámbitos que ya no están separados.

Quizá donde más sonora se haga esta transición sea en la cadena de nombres que se han dado a la intervención más difícil en esta situación, dado que de hablar de "no iniciar o retirar la reanimación", equiparándolos entre sí (Aguayo et al. 20I I: $68)$, se pasa a hablar consecutivamente de:

- "limitar el esfuerzo terapéutico" (LET) (Grupo de trabajo sobre LET de SENeo, 2002), con lo que se deja de nominar la defensa de la vida por encima de todo y además se abre la puerta a la intervención de la familia (Gálvez, 2006);

- "ajuste/adecuación del esfuerzo (diagnóstico) y terapéutico” (AE(D)T), que remarca la cantidad de esfuerzo y trabajo que conlleva y hace de la familia no solo agente sino también receptora de los cuidados (Tejedor et al., 2013; García-Muñoz et al., 20I4);

- "cuidados paliativos perinatales" (Balaguer et al., 20 2; Samaniego y Caserío, 2015) $)^{9}$ que se presenta ya como un concepto y una perspectiva

9. Recuérdese que en 20I4 el Consejo Interterritorial del SNS aprobó promover en nuestro país unidades que aplicaran este tipo de cuidados a niños, aunque con poco éxito todavía. 
más 'comprensiva', 'integradora', 'integral' e 'interdisciplinar' que abarca distintos agentes (pacientes, familiares y profesionales), aspectos (físicos, psicológicos, sociales, espirituales, etc.) y, por tanto, desmonta la disgregación de los diferentes ámbitos implicados, superando el aislacionismo mentalista cartesiano en la concepción de la VPE.

2. Al pasar al análisis de los discursos de familiares y de profesionales producidos en nuestra investigación ${ }^{\mathrm{Io}}$, que no tienen la contención ni el control de las publicaciones, va a ser algo más fácil apreciar que estamos en transición hacia una perspectiva más compleja e interconectada de la viabilidad en la prematuridad extrema.

El análisis sociodiscursivo muestra que estos discursos se organizan en torno a tres núcleos temáticos: el establecimiento de (los límites de) la viabilidad, las mediaciones tecnológicas y procedimentales y la "gestión" de la vida y la muerte. En relación con cada uno de estos nudos hemos agrupado, por un lado, los discursos médicos con los de otros profesionales, pero diferenciándolos de estos últimos, y, por otro, los discursos de los familiares.

Respecto a la cuestión de establecer la viabilidad, lo primero que llama la atención es que los discursos médicos, en especial los de los jefes de Unidades/ Servicios de Neonatología, se centran en destacar cómo la zona de penumbra -que algunos extienden hasta las 25 semanas de EG- y el límite de la VPE han variado

Io. Queremos dejar constancia aquí de nuestro agradecimiento a las muchas familias y profesionales que se han implicado en el desarrollo de nuestra investigación. No siempre ha sido fácil ni para las familias ni para los profesionales entrevistados compartir situaciones tan complejas y emociones tan intensas como las que se producen en torno a la supervivencia o no de hijos y pacientes tan pequeños y vulnerables. En adelante recogeremos algunos de sus testimonios pero, tanto por respeto como por secreto profesional, no daremos datos sobre su identidad personal. a lo largo del tiempo (I975, I990, 2004, etc.) y de unos países a otros (quizás como una forma de tomar distancia hacia un proceso en cuyos resultados están directamente implicados). En cambio, el resto de profesionales sanitarios parecen supeditar inmediatamente la VPE a otras cuestiones como la existencia de recursos dentro y fuera de las UCIN o la excesiva medicalización (la decisión queda en manos del médico y en base a capacidades biotecnológicas), que tiende a excluir al personal de enfermería y a los padres, no facilita los "abordajes multidisciplinares" y a menudo soslaya el dolor que produce a corto y largo plazo toda esta situación, haciéndolo más difícil de comprender por muy cerca que se esté de él, como se dice reiteradamente en el grupo de discusión hecho con enfermeras. Estos otros profesionales parecen, así, más proclives a percibir la complejidad de la VPE y su irreductibilidad a criterios estrictamente médicos o a decisiones meramente éticas.

Sin embargo, una mirada más atenta permite apreciar que la concepción que tiene el personal médico de nuestro país sobre los límites de la viabilidad se va acercando a una perspectiva similar a la de otros profesionales ${ }^{\text {II }}$. Así se muestra ya en la Declaración de Barcelona sobre los Derechos de la Madre y del Recién Nacido ${ }^{12}$, en las publicaciones

II. Una perspectiva que se ha visto refrendada en el I 2 th World Congress of Perinatal Medicine, celebrado en noviembre de 2015 en Madrid. En la sesión dedicada en dicho Congreso a los límites de viabilidad, dos de las principales voces internacionales sobre esta cuestión, la de los decanos $\mathrm{O}$. Saugstad (Noruega) y A. Fanaroff (EE.UU), que dedicaron sus ponencias a un metaanálisis de distintos estudios y normativas, llegaron a la misma conclusión: en principio, está claro que con $\leq 22$ de EG no hay beneficio en aplicar maniobras de resucitación, pero si lo hay con $\geq 25$ semanas; en cambio, no está claro qué decisión tomar entre las 23 y 24 semanas, es más, probablemente nunca lo estará ni tampoco sería deseable que hubiera un protocolo cerrado, por lo que se trataría de atender a la madurez específica del bebé y plantear una decisión colegiada que integre otras voces, especialmente la de los padres y la del personal de enfermería.

I2. Promovida en 200I por el 5 th World Congress of Perinatal Medicine, que en su apartado Io plantea: "No debe intentarse hacer sobrevivir a un recién nacido cuando su inmadurez es 
relacionadas con esta cuestión (Iriondo et al., 201 i; Sánchez Luna, 20I4; GarcíaMuñoz et al., 20I4) o cuando nuestros entrevistados enumeran los medios que han ido ampliando y determinando la viabilidad (apertura de UCIN, respiradores específicos, alimentación parenteral, catéteres epicutáneos, surfactantes, corticoides prenatales, resonancias y ecografías cerebrales, lactancia materna, método canguro, etc.). Esta enumeración permite percibir cómo la viabilidad emerge constituida por procedimientos, aparatos, fármacos, etc., y que, de hecho, como afirma el jefe de una importante UCIN, antes de la década de los 90, los prematuros extremos "se morían a las dos horas de vida y no te daba tiempo a plantear nada" y no es sino hasta comienzos de este siglo cuando las condiciones biotecnológicas y sociales permiten y llevan a plantearse la LET. Todo lo cual, unido a la remisión continua que se hace en el discurso a las diferentes condiciones de la viabilidad de la prematuridad extrema que hay en los países desarrollados (Holanda, Suecia, EE.UU, etc.), esto es, al hecho de que la organización sociosanitaria determina unas u otras condiciones a la VPE, viene a reiterar la complejidad de esta y su inserción en la realidad políticosocial.

El admitir que la VPE va más allá de criterios y pronósticos médicos y de consideraciones éticas se hace ya inevitable cuando distintos profesionales médicos achacan una y otra vez el aumento de la prematuridad al retraso en la edad de maternidad, que lleva a la utilización de tratamientos de infertilidad y/o de reproducción asistida, con los consiguiente partos múltiples "en los que, para mantener la situación de los recién nacidos y de la madres se favorecen los partos antes de

superior al límite inferior de viabilidad. En estos casos los padres deberán estar informados y participar en las decisiones prenatalmente, siempre que ello sea posible. Se tendrá en cuenta el ámbito geográfico, social y sanitario del lugar de nacimiento". tiempo" ${ }^{13}$ y más aún cuando nuestros entrevistados suman otros factores coadyuvantes como el aumento de la tensión laboral de las madres, especialmente las inmigrantes con embarazos poco controlados, o cuando se recuerda la cadena de instancias sociopolíticosanitarias que inciden en el establecimiento e implementación de los programas de actuación: legislación estatal, Consejo Interterritorial, Consejerías de Salud, gerencias de hospital, infraestructuras hospitalarias, etc. Basta con sumar todos estos planteamientos, que son ampliamente compartidos, para ver emerger una mirada que sitúa la VPE en medio de nuestras formas más generales de gestionar la reproducción y de controlar la vida y la muerte, esto es, que la sitúa como un hecho biopolítico fundamental.

También en el discurso de los padres encontramos esta perspectiva más compleja cuando vinculan la VPE a los avances médicos, a las dolorosas vivencias familiares, a los recursos externos disponibles y, sobre todo, al proceso de decisión de implementar la AET. En este sentido, es reseñable que, aunque los padres recuerden su participación en este proceso como una experiencia muy dura, donde se sentían inseguros, solos, impotentes, llenos de dolor y de emociones contradictorias, terminan admitiendo que cuando "me lo pintaron tan negro" no quedó más remedio que decir "que sea lo que dios quiera, pero si me lo pides a mí, a pesar de que sea mi hijo, no le hagas sufrir", como reconoce uno de los padres entrevistado. Lo cual es coherente con que también en el grupo de discusión que hicimos con madres y padres

I3. Si atendemos a los resultados cuantitativos de nuestro estudio, hay que señalar que el $72,2 \%$ de las madres de nuestra muestra tenían más de 30 años cuando nació el hijo; que partos múltiples lo fueron el $37,33 \%$ del total, y que el $24,17 \%$ del total de las encuestadas había utilizado algún tipo de tratamiento de infertilidad y/o de reproducción asistida, cifra que se eleva al $38 \%$ en el caso de las madres de nuestra muestra que tenían más de 35 años al nacer el hijo. En cualquier caso, es importante poner de manifiesto que el conjunto de condiciones causales de la prematuridad es muy amplio. 
se reitere que se encontraron situados ante la incertidumbre de un diagnóstico que decía: "es que no sabemos, es que es muy pequeño, pueden pasar muchas cosas". Y, sobre todo, es coherente con el amargo lamento de la madre de una prematura de I 2 años, con graves discapacidades cerebrales y motoras, que se queja del afán de los médicos por tirar para adelante como fuera y de haberla informado demasiado tarde (con I 8 meses de edad de su hija) de lo que se le venía encima, "porque dejarlos marchar no es malo", y que luego, más calmada, afirma: "yo veo a muchos papis que habían estado mucho más preparados para pasar el duelo que de lo mal que lo están pasando cuidándoles”.

Aunque los profesionales, especialmente de enfermería, son sensibles a la angustia que asalta a los padres ante esa "codecisión", y más desde que la apertura 24 horas de muchas Unidades los sitúa más cerca de su quehacer diario, su discurso apunta sobre todo al hecho de que en relación con la VPE se está todavía en transición, en una situación de oscilación entre el atribuir todo el control y la responsabilidad de la decisión al médico y el asumir que esta debe ser compartida con los padres y, en menor medida, con el personal de enfermería. Por su parte, muchos médicos, especialmente los jefes de servicio, aunque admiten la conveniencia de la codecisión, especialmente en la zona de penumbra, la rebajan rápidamente aludiendo a que no hay que poner la carga de una decisión tan difícil en los padres, o a que legalmente el único que tiene que firmar es el médico diciendo que hay acuerdo, o a que el objetivo es convencer a los padres, que "entiendan y acepten que eso es lo mejor para su hijo", pero la decisión "la toma el médico", es "el responsable último" ${ }^{\mathrm{I}}$.

I4. Es curioso que quien hace esta afirmación ha visto una decisión médica revocada por el comité de ética a instancia de los padres, sirviendo así de ejemplo de cómo las nuevas prácticas van por delante de lo que se piensa/dice y hacen cada vez más difícil seguir forzando la reducción de la complejidad de la VPE.
Llegamos así al último gran nudo detectado en estos discursos, el concerniente a la "gestión" de la vida/muerte en la prematuridad extrema, en el que, como no puede ser de otra manera, la mayor resonancia aparece en las voces de los padres. Sus palabras muestran la existencia de toda una serie de mecanismos psíquicos para afrontar esa "gestión” para, por ejemplo, hacer frente al hecho de que un hijo prematuro extremo viva a la vez que se intenta asumir la muerte de su hermano y se haya de convivir durante un largo tiempo con la posibilidad del fallecimiento del hijo prematuro superviviente. Son distintos y variados los recursos que los padres utilizan a la hora de enfrentar estas situaciones, tales como el humor para desdramatizar y distanciarse del "shock de ver por primera vez a tu hijo conectado a cables (...), aquello ni es un niño ni es nada, es como una masita" y describen al hijo como "un tamagochi pequeñín"; o la racionalización para intentar comprender lo que viene pasando, como cuando se ven a sí mismos y a sus hijos "siempre en tiempo de descuento". En definitiva, son mecanismos que traslucen que la vivencia parental de esta difícil situación se estructura siempre desde la ambivalencia y el sentir de que se está en el filo entre la vida y la muerte cuando nacen niños con tan poca edad gestacional: “ $y$, de hecho, la gente no sabe si llamarte o no llamarte, si darte la enhorabuena o el pésame", como quedó dicho en el grupo de madres y padres.

En el caso de los médicos, el discurso sobre este tipo de "gestión" apela básicamente al establecimiento de la viabilidad o no del recién nacido en el sentido ya comentado. Sin embargo, si nos centramos en el cambio sucedido a principios de este siglo respecto a los casos conflictivos, los médicos plantean que antes, como "no teníamos ventanas para ver cómo estaba el cerebro de esos niños", una vez que se decidía sacar al niño adelante se le sacaba "hasta que se moría"; ahora, en cambio, el protocolo lleva al personal médico a consensuar la decisión con los padres y, en 
los casos en los que no hay acuerdo, a remitir la decisión al comité de ética del hospital. Así presentado este proceso podría inducir a pensar que lo único que se ha hecho es complementar el diagnóstico clínico con un juicio ético elaborado por unos expertos. Pero no conviene dejarse engañar por las etiquetas. El “comité de ética” en sí mismo es ya un ensamblaje, un grupo multidisciplinar formado por profesionales de distintos ámbitos, algunos ajenos incluso al medio sanitario, que representan también las normas e intereses de la sociedad. A lo cual hay que añadir todo lo que está activamente implicado en esas decisiones liminares y que aparece explícitamente en el discurso mismo de los facultativos, desde la tecnología (por ejemplo, las resonancias que abrieron "esas ventanas"), a las autoridades sanitarias, que con normativas como la "regionalización" regulan qué tipos de Unidades -de qué nivel asistencial- deben atender a los neonatos de $\leq 25$ semanas, pasando por la formación sobre mortalidad que reciben los residentes, los protocolos que se establecen a partir de 2005 , hasta la codecisión con los padres y el cambio general de la cultura sociosanitaria. Tanto esta complejidad como las comprensibles resistencias de los distintos profesionales se ven muy claras en las siguientes palabras del jefe de una importante UCIN de nuestro país:

\footnotetext{
"Digamos que el cuidado médico tiene su magia también, y su presencia. Y entonces, pues los médicos, en algún momento que te contradiga un padre y que diga que no está de acuerdo, hace unos años eso era impensable ${ }^{15}$. (...) Entonces, yo creo que por una parte perder esa presencia, entre comillas, pues cuesta y necesita un aprendizaje, y necesita una reflexión, y necesita un ambiente social. (...) Y con la
}

I 5. Resonancias de esta afirmación se escuchan en el caso de unos padres que tuvieron tres hijos prematuros, uno de los cuales murió, cuando relatan que hace I 4 años padecieron la brusquedad de un médico que para convencer a la madre de que se retirara la leche le dijo "total, para un cuarto de gramo que has tenido" y afirman que "era un señor mayor, como prepotente". enfermería pues pasa algo parecido. Digamos que la enfermera es la dueña del niño. Entonces, si están los padres, pues los padres son una invasión y son un incordio, entre comillas. Porque te vigilan, te controlan, te preguntan, te dicen, te... Entonces, tú que eres la que sabe, o el que sabe, y eres la importante y la experta, pues tienes que prestar atención y tienes que admitir que alguien te ponga en entredicho. Entonces, es un cambio de mentalidad y un cambio de cultura que cuesta" (la cursiva es nuestra).

Esta nueva cultura sociosanitaria, que tiende a reequilibrar las posiciones, voces e intereses de los distintos agentes, se genera y sostiene no solo con la implantación de normativas o protocolos específicos, innovaciones tecnológicas o modificaciones procedimentales, sino también por el creciente empuje de las asociaciones de pacientes y familiares que plantean sus derechos (Wehling et al., 20I 5 ) y por ser parte importante en una redefinición general de las fronteras entre la vida y la muerte, de lo que al respecto es posible o imposible, deseable o indeseable, aceptable o inaceptable (Rose, 2005; Rabinow y Rose, 2006). Como dijimos, los conflictos de intereses por (re)definir los mapas de (im) posibilidades y los valores, son cuestiones políticas, en este caso biopolíticas; es más, son el núcleo de lo político en las democracias plurales y desarrolladas (Mouffe, I999). Aunque quizá, dado su elevado componente tecnológico (en lo mecánico, lo informacional y lo comportamental), habría que hablar mejor de tecnobiopoder.

\section{Conclusiones}

El análisis de los principales discursos involucrados en la atención a la VPE en nuestro país señala que se viene produciendo una transición en los últimos años hacia una nueva concepción de la VPE que resalta su complejidad, su multidimensionalidad y su naturaleza no solo biosocial sino también biopolítica. 
Analizar los discursos que explican qué es y cómo se gestiona la viabilidad en la prematuridad extrema nos ayuda a reconstruir este complejo prisma desde las distintas caras que presenta y a superar las diferentes tendencias a minimizar o amplificar la cuestión (Saigol y Doyly, 2008; Amy, 2010). También permite apreciar que la concepción canónica que disgrega la viabilidad en la prematuridad extrema poniendo en un lado los avances médicos, en otro los argumentos éticos y al recién nacido aislado de su entorno constitutivo, se ha ido diluyendo en favor de una perspectiva que asume la complejidad y la multidimensionalidad de la viabilidad y la hace irreductible a un pronóstico estadístico, a un algoritmo o a la decisión de un comité de ética al evidenciar que necesariamente involucra una negociación complicada y dinámica entre los muchos agentes e intereses implicados: del recién nacido a la distribución social de recursos sanitarios, el personal médico y de enfermería, los padres, protocolos y tecnologías implementadas, legislaciones diversas, etc. Las nuevas prácticas implantadas en las UCIN parecen ser un factor clave para explicar la transición que se vive actualmente en la concepción de la viabilidad en la prematuridad extrema en España, por cuanto la redistribución de las posiciones ocupadas que implican dichas prácticas rebajan la soledad, la responsabilidad y el poder del personal médico en la toma de decisiones y socializan ${ }^{16}$ la viabilidad en la prematuridad extrema.

I6. Ahora bien, al afirmar la naturaleza social de la VPE no nos limitamos a llamar la atención sobre su obvia inclusión en una serie de relaciones humanas, sino que apuntamos a la necesidad de considerarla como una componenda de relaciones y flujos materiales, emocionales e informacionales, de los cuales algunos pasan por o son movilizados por seres humanos (médicos, bebés, madres, padres, enfermeras, familiares, etc.), tanto en el nivel consciente o discursivo como en el corporal o emocional, mientras otros dependen de programas de acción, mediaciones tecnológicas, condiciones espaciotemporales, etc. Se hace así manifiesto el complejo y dinámico ensamblaje de múltiples ingredientes (humanos y no humanos) que, entretejiendo y actualizando corporeidades, recursos, protocolos, trasformaciones, espacios, etc., no solo encauzan la configuración y desarrollo de la prematuridad extrema, incluyendo su viabilidad, sino que también la actualizan y constituyen de maneras diversas. Otra razón para hablar de tecnobiopolítica.
Es cierto que el haber admitido la relevancia de las consideraciones éticas para resolver cuestiones que atañen a la viabilidad en la prematuridad extrema ha abierto las decisiones clínicas a otras voces y argumentos, rompiendo el aislamiento del facultativo y ampliando la perspectiva, $y$, en este sentido, ha sido un paso fundamental en este proceso. Sin embargo, a la vista de los desarrollos biomédicos y de los cambios sociales, resulta insostenible restringir la cuestión a un debate (también aislado) entre expertos, silenciando las voces e intereses de otros agentes constituyentes de esa viabilidad como son los familiares, las autoridades sanitarias, el proyecto vital de las mujeres, la cultura sociosanitaria o incluso las implementaciones tecnológicas, procedimentales y de apoyo institucional (Lemke, 20I I). Nos encontramos en una transición que parece llevarnos del momento ilustrado de una bioética disgregada y reduccionista, que concibe al individuo como preestablecido y aislado, a una nueva concepción propia del espacio más complejo, disputado y abierto de la biopolítica de las sociedades contemporáneas, que sitúa al individuo "en la interfaz de lo social y lo tecnológico” (Lash, 2002).

Por todo ello, parece que afrontar actualmente la VPE en toda su complejidad no pasa tanto por complementar la mirada médica con la ética o bioética, discurso de especialistas sobre el valor de la vida, cuanto por situarla en la (tecno)biopolítica, esto es, en la implementación abiertamente disputada de distintas técnicas, agentes e intereses orientados a producir y controlar la vida y las conductas. 


\section{Referencias bibliográficas}

Aguayo, J. et al. (20I I): El final de la vida en la infancia y la adolescencia: aspectos éticos y jurídicos en la atención sanitaria (en línea). $<$ http://www.secpal.com/\% ${ }_{5}$ CDocumentos $\%{ }_{5} \mathrm{C}$ Blog\% 5 Carchivo_304.pdf>, acceso 6 de mayo de 2016.

Alonso, E. et al. (20I2): "Mortalidad infantil en un hospital de nivel terciario. Limitación de esfuerzo terapéutico, correspondencia clínicopatológica y precisión diagnóstica”. Anales de Pediatría, 76 (6): 343-349.

Amy, L. et al.(2010): "Biological and Environmental Predictors of Behavioral Sequelae in Children Born Preterm". Pediatrics, I 25 (I): 83-89.

Balaguer, A. et al. (2012): "The model of palliative care in the perinatal setting: a review of the literature". BMC Pediatrics, I2: 25.

Cabero, L. (2009): "Declaración de la Comisión de Bioética de la Sociedad Española de Ginecología y Obstetricia sobre la interrupción del embarazo". Progresos de Obstetricia y Ginecología, 52 (I): 67-8.

Caserío, S. (20I4): Decisiones en torno a la LET en la Unidad Neonatal (en línea). <http:// es.slideshare.net/laianı/proyectohera-caserionueva>, acceso 9 de abril de 2015.

Cole, T.J. et al. (2010): “The PREM score: a graphical tool for predicting survival in very preterm births". Arch Dis Child Fetal Neonatal, 95: I4-I9.

Foucault, M. (I976): Historia de la sexualidad I. La voluntad del saber, Buenos Aires: Siglo XXI.

Gálvez, A. (2006): Muerte alrededor del nacimiento. Creencias, sentimientos y vivencias. Una perspectiva de los profesionales de los cuidados (Tesis doctoral). Universidad de Alicante.

García P. et al. (2013): "Evolución a los 2 años de edad corregida de una cohorte de recién nacidos con peso inferior o igual a I.500 g de los hospitales pertenecientes a la red neonatal SENI 500”. Anales de Pediatría, 79: 279-287.
García-Muñoz, F. et al. (20I4): “Morbimortalidad en recién nacidos al límite de la viabilidad en España: estudio de base poblacional". Anales de Pediatría, 80 (6): 348-356.

Gladys, E. et al. (2004): "El prematuro en cuidado intensivo neonatal. ¿Cuándo es el momento de decir no más? Reflexión bioética en torno a la limitación del esfuerzo terapéutico". Revista chilena de pediatría, 75 (2): I 8I-I87.

Gómez, C. (20I4): “Asociaciones de padres y seguimiento multidisciplinar de la gran prematuridad". Revista Española de Discapacidad, 2 (I): 203-2I3.

Gómez, C. et al. (20I4): "Dificultades biosociales de la gran prematuridad”, en Cairo, $\mathrm{H}$. y Finkel, L. (coords.): Crisis y cambio: Propuestas desde la Sociología. Actas del XI Congreso Español de Sociología: I229-I 238.

Grupo de trabajo de la SENeo sobre LET y Cuidados Paliativos en recién nacidos (2002): "Decisiones de limitación del esfuerzo terapéutico en recién nacidos críticos: estudio multicéntrico". Anales de Pediatría, 57 (6): 547-53.

Guzmán, J.M., et al. (20I I): Estudio comparativo de morbimortalidad de los $R N$ incluidos en la Red SENI 500 durante el periodo (2006-2010) (en línea). <http://www.se-neonatal.es/Portals/o/ SEN-I 500/Morbi-Morta_2006_20I0senI 500.pdf>, acceso I4 de abril de 2015 .

Hernández, N. et al. (2005): "Morbilidad a los dos años en niños con un peso al nacimiento menor de I.500 g.". Anales de Pediatría, 62: 320-327.

Instituto Nacional de Estadística (2008): Encuesta sobre Discapacidades, Autonomía personal y situaciones de Dependencia (en línea). <http:// www.ine.es/dyngs/INEbase/es/operacion.ht $\mathrm{m} ? \mathrm{c}=$ Estadistica_C\&cid $=\mathrm{I} 254736 \mathrm{I} 76782 \mathrm{Q}$ menu=resultados \&secc $=$ I 254736 I $947 \mathrm{I} 6 \& \mathrm{i}$ $\mathrm{dp}=\mathrm{I} 254735573$ I $75>$, acceso 20 de junio de 2016.

Iriondo, M. et al. (201 I): “Adaptación de las recomendaciones internacionales sobre 
reanimación neonatal 2010: comentarios". Anales de Pediatría, 75 (3): 203.eI-203.eI4.

Jiménez, R. y Molina, V. (2008) Bases éticas de la Neonatología. Decisiones de tratamiento selectivo en recién nacidos. Normas básicas de actuación (en línea). <https://www.aeped.es/ sites/default/files/documentos/54.pdf $>$, acceso I4 de abril de 2015 .

Lash, S. (2002): "Individualization in a non-linear mode”, en Becks, U. y Beck-Gernsheim, E. (eds.): Individualization. London: Sage.

Lemke, T. (20I I): Bio-Politics. An advanced Introduction, New York: New York University Press.

Manktelow, B. et al. (2013): "Population-Based Estimates of In-Unit Survival for Very Preterm Infants”. Pediatrics, I3 I (2): e425-432.

Moor, T. et al. (2012): "Neurological and developmental outcome in extremely preterm children born in England en 1995 and 2006: the EPICure studies". BMJ, 345: e796r. doi: Io.I I36/bmj.e796I.

Moreno, J.M. (20I 5): “Hidratación y alimentación en los recién nacidos: adecuación del esfuerzo terapéutico". Cuadernos de Bioética, XXVI (2): 24 I-249.

Mouffe, C. (I999): El retorno de lo político, Barcelona: Península.

Ochiai, M. et al. (2OI4): "Survival and Neurodevelopmental Outcome of Preterm Infants Born at 22-24 Weeks of Gestational Age". Neonatology, $\underline{105}(2):$ 79-84.

Organización Mundial de la Salud (2015) Nacimientos prematuros. Nota descriptiva $N^{\circ}{ }_{3} 63$ (en línea). <http://www.who.int/ mediacentre/factsheets/fs $363 /$ es $>$, acceso 27 de enero de 2016.

Pi-Sunyer, M.T. (20I3): Apoyo Emocional a los profesionales de las Unidades neonatales.
Comunicación presentada en el XXIV

Congreso de Neonatología y Medicina

Perinatal, Barcelona, España.

Rabinow, P. y Rose, N. (2006): "Biopower Today". BioSocieties, I: I95-2I7.

Raspall, F. y Royes, A. (coords.) (2009): Documento sobre la limitación del esfuerzo terapéutico en las unidades de neonatología, Barcelona: Observatori de Bioètica i Dret.

Rose, N. (2005): The politics of life itself: biomedicine, power, and subjectivity in the twenty-first century, Princenton: Princenton U.P.

Saigol, S. y Doyly, L.W. (2008): “An overview of mortality and sequelae of preterm birth from infancy to adulthood". Lancet, 37I: 26I-269.

Salguero, E. (2013): Recién nacido gran inmaduro en el límite de viabilidad. Comunicación presentada en el XXIV Congreso de Neonatología y Medicina Perinatal, Barcelona, España.

Samaniego Fernández, M. y Caserío Carbonero, S. (201 5): "Neonatología: cuidados al final de la vida", en Astudillo, W. et al. (eds.): Medicina Paliativa en niños y adolescentes. San Sebastián: Paliativos sin Fronteras.

Sánchez Luna, M. (20I4): "Limite de viabilidad en la actualidad". Anales de Pediatría, 80 (6): 346-347.

Tejedor Torres, J.C. et al. (2013): "Recomendaciones sobre toma de decisiones y cuidados al final de la vida en neonatología”. Anales de Pediatría, 78 (I): I90.er-I90.er4.

Wehling, P et al. (2015): The Public Shaping of Medical Research: Patient Associations, Health Movements and Biomedicine, London: Routledge.

Weir, L. (2006): Pregnancy, Risk and Biopolitics, London: Routledge. 